\title{
Exfoliative Toxin Mediated Staphylococcal Scalded Skin Syndrome: A Review
}

\section{IJCRR}

Section: Healthcare

Sci. Journal Impact

Factor: 6.1 (2018)

ICV: 90.90 (2018)

(c) (i) (8)

Copyright@IJCRR

\section{Gayathri Sunil' ${ }^{1}$ Meghana Vinod ${ }^{2}$, Roshni PR ${ }^{3}$}

'Student, Pharm D PB, Department of Pharmacy Practice, Amrita School of Pharmacy, Amrita Vishwa Vidyapeetham, Kochi-682041, Kerala, India; ${ }^{2}$ Student, Pharm D PB, Department of Pharmacy Practice, Amrita School of Pharmacy, Amrita Vishwa Vidyapeetham,

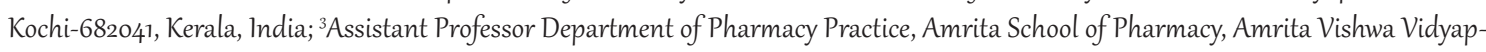
eetham, Kochi-682041, Kerala, India.

\section{ABSTRACT}

Staphylococcal scalded skin syndrome (SSSS) is a rare dermatological condition associated with extensive desquamation by toxicogenic strains of staphylococcus aureus. The two known serotypes of exfoliative toxin namely A and B are responsible for this disease entity. Haematogenous dissemination of toxin cause extensive epidermal damage at sites distant to the infectious site. When considering all age ranges, SSSS is more prevalent in infants and children below 5 years but can occur at any age. Initially, symptoms include fever, malaise, dehydration followed by the tenderness of the skin with erythematous rash, flaccid blisters and bullae which may rupture on pressure, leaving superficial crusts. Despite a well-known clinical aspect, several cutaneous diseases may be mistaken for SSSS. Therefore, rapid and definitive histopathological evaluation of the biopsy is essential to confirm the diagnosis. The prognosis is improved by adequate therapy, that is, aggressive fluid replacement, nutritional support and optimal antibacterial policy. Antibiotics covering staphylococcus are the preferred treatment as the disease is caused generally by a single organism, S. aureus, mainly belonging to phage group II, and which include Nafcillin or Oxacillin for methicillinsensitive Staphylococcus aureus (MSSA). If cultures are positive for MRSA, vancomycin should be used. Healing occurs within 10 to 14 days without scarring. This article aims to provide an overview of our current understanding of SSSS epidemiology, clinical features, pathogenesis, differential diagnosis and management.

Key Words: Epidermal damage, Erythematous rash, Antibiotics, infants, Staphylococcus aureus

\section{INTRODUCTION}

Staphylococcal scalded skin syndrome (SSSS) is a potentially fatal disease, commonly caused by pathogenic toxins of S aureus affecting the superficial layers of the epidermis ${ }^{1}$. Ritter's disease, Ritter von Ritterschein disorder (in neonates), bullous impetigo, are synonyms of SSSS. ${ }^{2}$ The disease primarily affects neonates and small children with immunodeficiency, under the developed renal system which is inefficient at clearing the exotoxin and lack of antibodies against Exotoxins (A and B) have been suggested as reasons. ${ }^{3,4}$ It has also been described in adults with long term renal disease or renal failure, immunologic deficiency, and other chronic illnesses. ${ }^{5}$ Mortality is significantly higher in adult (from $40 \%$ to $63 \%$ ) as compared with children (below $10 \%$ ), despite optimal antibacterial therapy. Mortality in infancy is low $(4 \%){ }^{3}$

SSSS is considered as one of the serious dermatological condition with an estimated incidence ranges between 0.09 and
0.56 cases per 1 million persons. ${ }^{6}$ The two pathogenic toxins produced in SSSS are exfoliative (or epidermolytic) toxin A (ETA) and exfoliative toxin B (ETB). S aureus strains implicated in SSSS may produce ETA, ETB, or both toxins. ${ }^{7}$ The causative toxins disseminate systemically from a confined infectious site causing serious and widespread epidermal damage at sites distant to the infectious site. ${ }^{8}$

The form and severity of SSSS vary ranging from the confined infectious blisters to generalised SSSS affecting $90 \%$ or more of the skin surface area. ${ }^{9}$ The initial presentation of the syndrome may include non-specific symptoms such as fever, fussiness, malaise, tiredness, facial oedema and redness of the skin. Blisters with tender erythematous rash develop within 24 to 48 hours, where the irritable skin eruptions initially start on the face and flexures (groin, axillae, neck) with superficial abnormal reddening of the skin and fissures. Later as the disease progress, large fluid-filled bullae arise on the erythematous skin resulting in a wrin-

\section{Corresponding Author:}

Roshni PR, Assistant Professor Department of Pharmacy Practice, Amrita School of Pharmacy, Amrita Vishwa Vidyapeetham, Kochi-682041, Kerala, India; Email: roshnipr@aims.amrita.edu

ISSN: 2231-2196 (Print)

Received: 18.08 .2020
ISSN: 0975-5241 (Online)

Revised: 15.09 .2020
Accepted: 22.10 .2020
Published: 24.11 .2020 
kled appearance, that rupture quickly to produce erosion and leave an area of moist skin that soon becomes tender and painful. ${ }^{10}$ The development of an epidermal separation in the erythematous areas with minimum pressure (Nikolsky's sign) is a characteristic feature of SSSS. Nikolsky sign is positive when the mechanical pressure causes an epidermal detachment. Shedding or peeling of the outermost layer of the epidermis often in large sheets might be present and the extensive area of desquamation appear scalded. ${ }^{11}$ The average incubation period from infection to onset of symptoms ranges approximately between 1 to 10 days and it heals within 10 to 14 days without scarring. ${ }^{12,3}$ Complications associated with SSSS include secondary infections like cellulitis, septicaemia, pneumonia, scarring, Guttate psoriasis, Post-streptococcal glomerulonephritis, dehydration, electrolyte imbalance, and hypothermia. ${ }^{13}$ This review aims to discuss the role of toxins responsible for SSSS, including the pathophysiology, clinical features, diagnosis and treatment of SSSS.

\section{PATHOPHYSIOLOGY}

SSSS is an acute exfoliation of the skin induced by toxicogenic strains of $S$. aureus, with the most common subsets being phage group two strains 55 and $71 .{ }^{10,14}$ Less common causative strains include types 3A, 3B, 3C, and the ST121 strain. Similarly, other $\mathrm{S}$ aureus belonging phage groups (group 1 and 3) also have shown to induce SSSS. Isolates of phage group II S. aureus strains produce four types of toxic proteins. Of all the toxic proteins encountered, A, B, C, and D toxins were very likely to cause epidermal detachment (Nikolsky's sign) in neonatal mice. ${ }^{15}$

ET A and ET B are two different ET serotypes responsible for SSSS, where ET B is described as thermo-labile whereas ETA is demonstrated as heat stable. ET A is composed of 242 amino acid and has a molar mass of $26,950 \mathrm{kDa}$ whereas ET $\mathrm{B}$ is made out of 246 amino acid and have a molar weight of $27,274 \mathrm{kDa}$. ET A gene is codified on chromosomal DNA, whereas ET B is plasmid found. ${ }^{16,17}$ The epidermolytic toxin (ETA and ETB) caused by Staphylococcus aureus are serine proteases, capable of cleaving desmoglein 1, a glycoprotein responsible for keratinocyte-to-keratinocyte adhesion in the superficial epidermis of the skin. Cleavage of this glycoprotein leads to skin fragility and the formation of superficial blisters and erosions. Both ETA and ET B spread through the bloodstream and act particularly in the stratum granulosum of the epidermis, and result in the formation of superficial skin lesions. Dislodgement of the intact superficial epidermis by a shearing force surface is counted as one of the principal indications of the syndrome. ${ }^{18,11}$

The epidermolytic toxin is serine proteases that target and selectively hydrolyze the protein desmoglein-1 (Dsg1), a desmosomal cadherin responsible for the integrity of cell to cell adhesion in keratinocytes and mucosal epithelial cells. Desmoglein-1 maintains the integrity of intracellular junctions through its adhesive function and abundantly expressed in stratum granulosum of the epidermal layer. ${ }^{19}$ In the epidermis, Dsg2 was found in all desmosome-possessing tissues, including non-epithelial myocardium whereas Dsg3 is the targeted protein in the autoimmune blistering dermatosis, pemphigus Vulgaris and was essentially constrained to stratum spinosum and stratum basale. Dsg1 is the specific receptor for exfoliative toxin A cleavage and disrupt intercellular adhesion in keratinocytes. Cleavage of this glycoprotein leads to skin fragility and the formation of superficial blisters and erosions in SSSS. ${ }^{18,20}$

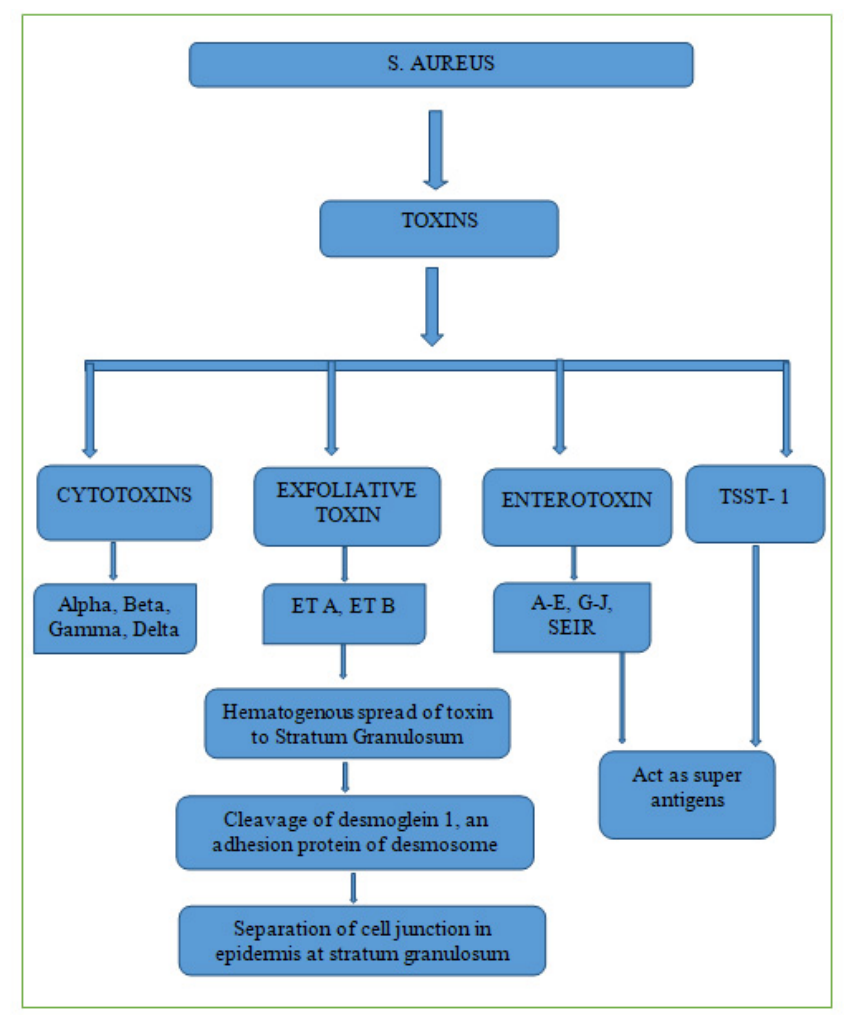

Figure 1: Pathophysiology of SSSS.

\section{INVESTIGATION AND DIAGNOSIS}

In general, SSSS diagnosis is achieved without difficulty, based on the signs and clinical presentation of typical small bullae and erosions. The analysis of both the localized and generalized forms of SSSS usually relies on the characteristic clinical appearance and few differential diagnoses. Isolation of Staphylococci producing exfoliative toxin from sites that are either colonized with bacteria or primary infection, such as the conjunctiva, nasopharynx, umbilicus, diaper area or suspect skin lesions often supports the diagnosis of $\mathrm{SSSS}^{21,22}$. Microbial culture of blood is typically negative, because the organisms are often non-invasive, especially in children, but can be 
positive in adults ${ }^{12}$. Fluid-filled blister in generalized SSSS appears to be sterile while the fluid in bullous impetigo yields a pathogen. ${ }^{23,24}$ Although not usually necessary, in some cases, a skin biopsy, in which a skin lesion is removed and studied under a microscope, may be performed which helps to rule out other bullous dermatoses, and can reveal non-inflammatory superficial cleavage or separation of the epidermis at the granular layer, which is indicative of the disorder. Skin biopsy is one of the important diagnostic tests for definitive diagnosis of SSSS, especially if the case is refractory. ${ }^{25}$

Phage typing of the S. aureus ETs or polymerase chain reaction (PCR) serum test for exfoliative toxins firmly supports SSSS diagnosis, even though other phage types have been shown to generate the same clinical picture. If the outbreak occurs and further confirmation is needed, bacteria swab of s. aureus may be sent for phage typing to the Public Health Laboratory (PHL), UK, which is not routinely available in hospital laboratories. ${ }^{26}$

PHL can attempt to test for organism producing ETs using immunological Ouchterlony method, for further confirmation of the diagnosis. But this method is unsuitable for broad sample batch processing, as it lacks sensitivity and specificity and is, therefore, useful retrospectively. ${ }^{23,26}$ The staphylococcal scalded skin syndrome may often mimic another widespread blistering disease, including toxic epidermal necrolysis, Stevens-Johnson Syndrome, bullous impetigo, pemphigus Vulgaris, epidermolysis bullosa, bullous mastocytosis, bullous pemphigoid, scalding thermal burn, Kawasaki disease, scarlet fever, and peeling skin syndrome. SSSS must therefore be differentiated from other intraepidermal conditions as it could encourage secondary infection with potentially fatal outcomes. ${ }^{22,23}$

Toxic Epidermal Necrolysis (TEN), also called as Lyell's Syndrome is a type of severe adverse immunological skin condition most often triggered by a certain drug reaction or their metabolites, characterized by macular exanthema, haemorrhagic erosions, erythematous eruption, mucosal lesions, blisters with a positive Nikolsky sign and may lead to multi-organ damage. ${ }^{27} \mathrm{TEN}$ can present in any age group but occurs more frequently in women, HIV-Infected patients, and the elderly. SSSS, Multiform exudative erythema, Toxic epidermal necrolysis, and Stevens-Johnson syndrome belongs to the same disease spectrums. As in SSSS, fragile blisters and positive Nikolsky sign are also seen in TEN/SJS, even though the cause and development of both disorders are different. However, eroding TEN/SJS lesions can be histologically distinguished from SSSS lesions. ${ }^{28}$

Toxic epidermal necrolysis can be quickly distinguished from SSSS by examining a frozen section of a biopsy specimen showing a superficial epidermal blister in scalded skin syndrome and a sub-epidermal blister in toxic epidermal necrolysis with necrotic keratinocytes. There are several features in common between SSSS and Bullous Impetigo. Nevertheless, SSSS skin lesion is larger compared to Bullous Impetigo, S. aureus is less commonly isolated and less inflammatory infiltrate in the skin lesions. ${ }^{29}$

Recently established diagnostic tests for exfoliative toxins of SSSS include enzyme-linked immunosorbent assays (ELISA), serological methods of gel immune precipitation(Mancini test), radioimmunological assays (RIA), gene sequence detection by DNA hybridisation as well as polymerase chain reaction(PCR) is used to detect causative toxin from suspected strains of the causative organism. ${ }^{26}$

\section{TREATMENT}

SSSS can be managed by the eradication of causative organism through appropriate antibiotics and supportive care to promote healing, reduce discomfort, and minimize complications. Until cultures are available for guiding therapy, Prompt empirical therapy with anti-staphylococcal antibiotics such as Nafcillin, Oxacillin, or Fucloxacillin for methicillin-sensitive Staphylococcus aureus (MSSA), is essential to eradicate primary infection. Other adjunctive agents include Cloxacillin plus tobramycin." or erythromycin . $., 3,23$

An increasing concern is an antibiotic resistance, with records of cases caused by methicillin-resistant S. aureus (MRSA). If the patient is critically ill and not responding to empiric treatment, MRSA should be considered. Vancomycin or linezolid are indicated if MRSA is suspected in areas with significant MRSA prevalence. ${ }^{30,31}$ Recommended therapies for SSSS include first and second generation Cephalosporins ${ }^{23}$. Clindamycin can be considered as a drug of choice for cutaneous staphylococcal infection due to its unique function as an inhibitor of bacterial toxin production in addition to bacteriostatic action and have excellent skin penetration. The retrospective study on antibiotic Sensitivity and Resistance patterns in the paediatric population with SSSS suggest that clindamycin can be used in combination with penicillinaseresistant penicillin rather than clindamycin monotherapy for empirical SSSS treatment until culture sensitivities are available for guiding therapy. ${ }^{32}$

Topical antibacterial agents such as Co-trimoxazole, Fusidic acid, Mupirocin, can also be considered as an adjunct for localised lesion treatment in infectious areas to eradicate the colonization of organism, which produce the toxins locally. Based on both experimental and clinical evidence the use of corticosteroid is contraindicated as it may worsen the disease. Potential carriers of toxin-producing strains must also be identified and treated to prevent cross-transmission, which may result in outbreaks. ${ }^{22,33}$ Due to enhanced systemic absorption by denuded skin, Silver sulfadiazine, which is still used in some burns unit, is not recommended for $\mathrm{SSSS}^{34}$. Intravenous immune globulin or plasma exchange had also 
been recommended to combat Staphylococcal scalded skin syndrome, but a recent study correlates its use with prolonged hospitalization. However, data are insufficient to confirm the efficacy of these therapies. ${ }^{35,36}$

For children with generalised SSSS, particularly neonates, general treatment strategies include supportive nursing care, adequate analgesia, proper wound care and prevention of dehydration is a critical component in management which result in quick recovery from SSSS. Adequate pain management therapy should be provided as the lesions are often painful. Careful monitoring should be ensured to prevent and/or treat evidence of secondary infection which may be fatal. With appropriate treatment and care the skin lesions usually heal rapidly over the next 7-10 days and complete resolution occurs in most cases within 2 to 3 weeks, with no permanent sequelae because the exfoliation is superficial and scarring is rare..$^{25}$

\section{Table 1: Antibiotic Therapy for SSSS}

\begin{tabular}{ll} 
Organism & Treatment \\
S.aureus MSSA & Naficillin \\
& $\begin{array}{l}\text { Oxacillin } \\
\text { Flucloxacillin }\end{array}$ \\
S.aureus MRSA & Vancomycin or linezolid \\
Topical antibiotic & Co-trimoxazole \\
& Fusidic acid \\
& Mupirocin \\
\hline
\end{tabular}

\section{FUTURE PERSPECTIVES}

Although SSSS is relatively uncommon and usually easily diagnosed on the clinical picture, developing new novel detection methods and improving existing techniques becomes a heightened priority for confirmation of SSSS. Developing a rapid, sensitive and specific diagnostic kit with simple tests to capture the level of toxin in serum or bullae, to be available within a short period will substantially alter the management of SSSS. It would allow a definitive diagnosis of SSSS and help in early detection and initiation of appropriate treatment regimen particularly among adult where morbidity and mortality rates are high. It also helps in the prevention of development of disease and minimises the risk of large outbreaks of infection by detecting carriers.

Another area that requires further research involves elucidation of the mechanism of action of the exfoliative toxins, for the design and development of antitoxin. ${ }^{25}$ Even though studies have shown that antitoxin antibodies will protect neonatal mice from lethal doses of ET, even if the antitoxin is administered late during the disease, there is a lack in clinical and experimental studies exploring within this area. Therefore research should focus on the establishment of structure and function of toxin that it may lead to the evolution of new antitoxin strategies shortly to prevent exfoliation.

\section{CONCLUSION}

Staphylococcus aureus is a widespread cutaneous pathogen that causes a broad spectrum of disease from mild to severe skin infections in humans. ${ }^{37}$ The two pathogenic toxin ET A and ET B are the causative agents of SSSS. The disease primarily affects neonates and children below the age of 5 , but occasional cases have been reported in adults with renal failure, immunologic deficiency, and other chronic illnesses. The initial presentation of SSSS shows non-specific symptoms such as fever, malaise, and lethargy followed by a generalized, tender erythematous lesion, widespread blistering and skin separation. Early diagnosis and prompt supportive therapy along with antimicrobial therapy improve clinical outcomes in patients with SSSS. ${ }^{23}$

\section{ACKNOWLEDGEMENT}

Authors would like to express gratitude towards the Department of Pediatrics, Amrita Institute of Medical Sciences, Kochi for providing necessary help for the diagnosis and treatment of SSSS

DISCLOSURES: The authors declare that there is no conflict of interest in this article content.

\section{Conflict of Interest: Nil}

Source of Funding: Nil

\section{REFERENCES}

1. Melish ME, Glasgow LA. The staphylococcal scalded skin syndrome: development of an experimental model. N Engl J Med 1970 May 14; 282(20):1114-9.

2. Melish ME. Staphylococci, streptococci and the skin: a review of impetigo and staphylococcal scalded skin syndrome. Semin Dermatol 1982; 1: 101-9.

3. Handler MZ, Schwartz RA. Staphylococcal scalded skin syndrome: diagnosis and management in children and adults. J Eur Acad Dermatol Venereol 2014 May 20; 28(11):1418-23.

4. Jeyakumari D, Gopal R, Eswaran M, Maheshkumar C. Staphylococcal scalded skin syndrome in a newborn. J Glob Infect Dis 2009 Jan-June; 1(1):45-7.

5. Cribier B, Piemont Y, Grosshans E. Staphylococcal scalded skin syndrome in adults: a clinical review illustrated with a new case J Am Acad Dermatol 1984 Feb; 30(2):319-24.

6. Mockenhaupt M, Idzko M, Grosber M, Schopf E, Norgauer J. Epidemiology of staphylococcal scalded skin syndrome in Germany. J Invest Dermatol 2005 April; 124(4):700-3.

7. Lamand V, Dauwalder O, Tristan A, Casalegno JS, Meugnier $\mathrm{H}$, Bes $\mathrm{M}$ et al. Epidemiological data of staphylococcal scalded skin syndrome in France from 1997 to 2007 and microbiological characteristics of Staphylococcus aureus associated strains. Clin Microbiol Infect 2012 Dec; 18(12): E514-24.

8. Kouakou K, Dainguy ME, Kassi K. Staphylococcal scalded skin syndrome in the neonate. Case Rep Dermatol Med 2015 June 8; 2015:901-68. 
9. Ladhani s, Newson T.A. A familial outbreak of staphylococcal scalded skin syndrome. Pediatr Infect Dis J 2000 Jan; 19(6): 578-9.

10. Patel GK, Finlay AY. Staphylococcal scalded skin syndrome: diagnosis and management. Am J Clin Dermatol 2003 Aug 21; 4(3): 165-75.

11. Moss C, Gupta E. The Nikolsky sign in staphylococcal scalded skin syndrome. Arch Dis Child 1998; 79: 290-3.

12. Gupta A, Jacobs N. Visual diagnosis: 2-week-old has a red, peeling rash. Pediatr Rev 2013 March; 34(3):e9-e12.

13. Mishra AK, Yadav P, Mishra A. A systemic review on staphylococcal scalded skin syndrome (SSSS): a rare and critical disease of neonates. Open Microbiol J 2016 Aug 31; 10: 150-9.

14. Haveman LM, Fleer A, Gerards LJ. Staphylococcal scalded skin syndrome in two very low birth weight infants. J Perinat Med 2003 June 1; 31(6):515-9.

15. Kondo I, Sakurai S, Sarai Y. Purification of exfoliatin produced by Staphylococcus aureus of bacteriophage group 2 and its physicochemical properties. Infect Immun 1973 Aug; 8(2):156-64.

16. Gemmell C G. Staphylococcal scalded skin syndrome. J Med Microbiol 1995 Nov 01; 43(5):318-27.

17. Freer JH, Arbuthnott J P. Toxins of Staphylococcus aureus. Pharmacol Ther 1982; 19:55-106.

18. Amagai M, Matsuyoshi N, Wang ZH, Andl C, Stanley JR. Toxin in bullous impetigo and staphylococcal scalded-skin syndrome targets desmoglein 1. Nat Med 2000 Nov; 6: 1275-7.

19. Getsios S, Huen AC, Green KJ. Working out the strength and flexibility of desmosomes. Nat Rev Mol Cell Biol 2004 April 01;5: 271-81

20. Payne A.S, Hanakawa Y, Amagai M, Stanley JR. Desmosomes and disease: pemphigus and bullous impetigo. Curr Opin Cell Biol 2004 Oct; 16(5):536-43.

21. Falk D K, and L E King. Criteria for the diagnosis of staphylococcal scalded skin syndrome. Cutis 1983 April 01; 31(4):421-4.

22. Leung AKC, Barankin B, Leong KF. Staphylococcal-scalded syndrome: evaluation skin, diagnosis, and management. World J Pediatr 2018 March 05; 14: 116-20.

23. Ladhani S, Joannou CL, Lochrie DP, Evans RW, Poston SM. Clinical, microbial and biochemical aspects of the exfoliative toxins causing staphylococcal scalded skin syndrome. Clin Microbiol Rev 1999 April; 12:224-42.

24. Elias P M, Fritsch P, Epstein E H Jr. Staphylococcal scalded skin syndrome Clinical Features, Pathogenesis, and Recent Microbiological and Biochemical Developments. Arch Dermatol 1977 Feb; 113(2):207-19.
25. Ladhani S. Recent developments in staphylococcal scalded skin syndrome. Clin Microbiol Infect 2001 June; 7(6):301-7.

26. Ladhani S, Evans RW. Staphylococcal scalded skin syndrome. Arch Dis Child 1998; 78: 85-8.

27. Lyell A. Toxic epidermal necrolysis: an eruption resembling scalding of the skin. Br J Dermatol 1956 Nov; 68(11):355-61.

28. Bastuji-Garin S, Rzany B, Stern RS, Shear NH, Naldi L, Roujeau JC. Clinical classification of cases of toxic epidermal necrolysis, Stevens-Johnson syndrome and erythema multiforme. Arch Dermatol 1993 Jan; 129(1):92-6.

29. John R S, Amagai M. Pemphigus, Bullous Impetigo, and the Staphylococcal Scalded-Skin Syndrome. N Engl J Med 2006 Oct 26; 355(17):1800-10.

30. Davidson J, Polly S, Hayes PJ, Fisher KR, Talati AJ, Patel T. Recurrent staphylococcal scalded skin syndrome in an extremely low-birth-weight neonate. AJP Rep 2017 April; 7(2):e134-7.

31. Jagadeesan S, Kurien G, Divakaran MV, Sadanandan SM, Sobhanakumari K, Sarin A. Methicillin-resistant Staphylococcus aureus colonization and disease severity in atopic dermatitis: A cross-sectional study from South India. Indian J Dermatol Venereol Leprol 2014 May 12; 80(3):229-34.

32. Braunstein I, Wanat KA, Abuabara K, Mcgowan K, Yan AC, Treat JR. Antibiotic sensitivity and resistance patterns in pediatric staphylococcal scalded skin syndrome. Pediatr Dermatol 2014 Aug 23; 31(3):305-8.

33. Ladhani S. Understanding the mechanism of action of the exfoliative toxins of Staphylococcus aureus. FEMS Immunol Med Mic 2003 Nov; 39(2):181-9.

34. Murray RJ. Recognition and management of Staphylococcus aureus toxin-mediated disease. Intern Med J 2005 Dec; 35(Suppl 2): S106-19.

35. Urata T, Kono M, Ishihara Y, Akiyama M. Adult Staphylococcal Scalded Skin Syndrome Successfully Treated with Multimodal Therapy Including Intravenous Immunoglobulin. Acta Derm Venereol 2018 Nov 1; 98(1):136-7.

36. Kato T, Fujimoto N, Nakanishi G, Tsujita Y, Matsumura K, Eguchi $\mathrm{Y}$ et al. Adult staphylococcal scalded skin syndrome successfully treated with plasma exchange. Acta Derm Venereol 2015 May; 95(5):612-13.

37. Mtsher AM, Aziz ZS. Estimation of Erythromycin and inducible Clindamycin Resistance in Staphylococcus aureus Isolated from Clinical Cases. Research J Pharm and Tech 2020; 13:29206. 